\title{
Cell-free DNA and chemoembolization in patients with liver metastases from colorectal cancer
}

\author{
ANDERS KINDBERG BOYSEN ${ }^{1,2}$, MARTIN JENSEN ${ }^{1,3}$, \\ DENNIS TØNNER NIELSEN ${ }^{4}$, FRANK VIBORG MORTENSEN ${ }^{5}$, BRITA SINGERS SØRENSEN ${ }^{2}$, \\ ANNI RAVNSB ÆK JENSEN ${ }^{1}$ and KAREN-LISE SPINDLER ${ }^{1,2}$ \\ Departments of ${ }^{1}$ Oncology and ${ }^{2}$ Experimental Clinical Oncology, Aarhus University Hospital, 8000 Aarhus C; \\ ${ }^{3}$ Department of Oncology, Aalborg University Hospital, 9100 Aalborg; Departments of ${ }^{4}$ Radiology \\ and ${ }^{5}$ Surgical Gastroenterology, Aarhus University Hospital, 8000 Aarhus C, Denmark
}

Received January 25, 2018; Accepted April 26, 2018

DOI: $10.3892 / 01.2018 .8925$

\begin{abstract}
Transarterial chemoembolization with irinotecan loaded beads (DEBIRI-TACE) represents an investigative treatment option for patients with metastatic colorectal cancer (mCRC). The present study examined DEBIRI-TACE with concomitant mFOLOFX6-bevacizumab as a first-line treatment for $\mathrm{mCRC}$ and explored the clinical value of circulating cell-free DNA (cfDNA). Patients with limited mCRC of the liver who had not been treated with chemotherapy received up to 4 biweekly DEBIRI-TACE treatments. The endpoints examined included the response rate, survival, toxicity and translational analysis. Due to toxicity and lack of feasibility, the study closed prematurely. Total cfDNA was measured with a direct fluorescent assay. Between December 2012 and February 2014, 14 patients underwent a total of 49 DEBIRI-TACE treatments. With a median follow-up of 1.7 years, the median progression free survival and overall survival (OS) were 240 days [95\% confidence interval (CI): 161-357] and 522 days (95\% CI: 174-1,054), respectively. The response rate was $50 \%$. Twelve patients experienced grade 3 toxicity or above. Dynamics of cfDNA showed biological variations in relation to therapy. To conclude, the present results indicated a response rate of $50 \%$ and median OS of 522 days for 14 patients with mCRC undergoing DEBIRI-TACE, but unacceptable toxicity and lack of feasibility with the applied schedule. The findings suggest that the level of cfDNA may be associated with the disease course, response to treatment and outcome. Trial registration: The European Clinical Trials database (EudraCT no. 2012-000987-11) at 05-14-2012.
\end{abstract}

Correspondence to: Dr Anders Kindberg Boysen, Department of Oncology, Aarhus University Hospital, Nørrebrogade 44, 8000 Aarhus C, Denmark

E-mail: andboy@rm.dk

Key words: colorectal cancer, liver metastases, chemo-embolization, cell free DNA

\section{Introduction}

Colorectal cancer (CRC) remains one of the most common malignant diseases with approximately 400,000 new cases word wide yearly (1). Close to $50 \%$ of patients diagnosed with CRC develop metastatic disease (mCRC) with the liver being the predominant organ affected (2). Surgical resection of liver metastases results in long term survival for about $40 \%$ (3) but only a minority of patients are eligible for resection. This has stimulated the interest in non-surgical local treatments including radiofrequency ablation (RFA) (4) and stereotactic body radiotherapy (SBRT) (5), both of which are established as standard of care.

Trans arterial chemo-embolization (TACE) was introduced in the 1980's by Yamada (6) with the rationale of embolic induced ischemia and necrosis and the delivery of chemotherapy with a minimal of systemic exposure. This technique was further refined with the introduction drug eluting beads (DEB) loaded with irinotecan (DEBIRI), and the first exploratory trial on DEBIRI-TACE for mCRC was published by Aliberti in 2006 (7). Today, the use of DEBIRI-TACE for mCRC is supported by several observational studies and two published randomized trials $(8,9)$, yet several unclarified issues regarding the optimal patient selection and technique still renders an unsettled place for DEBIRI-TACE in the overall treatment landscape for mCRC (10).

Cell-free DNA (cfDNA) constitutes all circulating DNA fragments, both mutant and non-mutant alleles, and can be detected and quantified in both healthy individuals, patients with various medical disorders and the highest values are seen in cancer patients (11). Since the first report of circulating nuclear acids in the blood stream in 1948 (12), emphasis on cancer patients was enhanced by Leon in 1977 (13). During the past decades, there has been a growing interest in the utility of cfDNA in various aspects of CRC management, both for early stage CRC (14) and a recent meta-analysis of 10 trials has shown a pronounced prognostic value of cfDNA for patients receiving systemic palliative chemotherapy (15).

In this study, we first report the results from a single center phase II study of DEBIRI-TACE plus intensified chemotherapy 
as first-line treatment for patients with liver limited mCRC. Second, we describe results of total cfDNA measured in plasma samples.

\section{Materials and methods}

Study design and ethics. This was an open-label phase II trial conducted at Aarhus University Hospital in co-operation between the Departments of Oncology, Radiology and Surgical Gastroenterology (Aarhus, Denmark). The aim was to investigate the feasibility, toxicity and efficacy of DEBIRI-TACE treatment with concomitant mFOLFOX6 as a first-line treatment for patients with liver limited mCRC. Patients were recruited from December 2012 to February 2014. The study planned to include 50 patients, but was stopped prematurely following the inclusion of 15 patients, by the decision of the study board and sponsor.

All patients included received a thorough verbal and written information and a signed consent was received prior to any protocol treatment. The protocol was approved by the National Board of Health (EudraCT no. 2012-000987-11), the Data Monitor Committee (J.nr. 2012-41-0370) and the Regional Ethics Committee (no. 1-10-72-306-12).

Patients and eligibility. Patients eligible for this study were newly diagnosed patients with liver limited mCRC not being candidates for any standard local treatment e.g., liver resection, RFA or SBRT. All potential candidates were systematically reviewed at a multidisciplinary hepato-pancreatic tumor board with experienced experts in radiology (including invasive radiology), oncology and liver surgery. Patients judged potentially eligible for local treatment in case of tumor shrinkage were allowed inclusion.

Key inclusion criteria were: Age $>18$ years, WHO Performance Status (PS) of 0-1, verified metastatic colorectal adenocarcinoma with no extra hepatic disease, less than $50 \%$ of the liver parenchyma involved and satisfactory organ function. Key exclusion criteria were: Presence of other malignant disease, prior chemotherapy within 6 months, trombo-embolic disease and factors not allowing for femoral artery access.

Treatments and procedures. The treatment scheme consisted of initially up to 4 DEBIRI-TACE treatments concomitant with systemic chemotherapy of mFOLFOX6 (5-Fluororacil, leucoverin, Oxaliplatin) + bevacizumab $24 \mathrm{~h}$ after embolization. Each lobe was treated twice with a 4 weeks interval, so patients with bi-lobar involvement received biweekly treatment alternating the lobes, allowing for up to 4 embolizations. Subsequent to the 4 DEBIRI-TACE treatments patients continued with systemic chemotherapy consisting of FLIRI (5-fluorouracil, leocoverin, Irinotecan) + bevacizumab for up to a total of 6 months treatment, which was the current standard of care.

Patients were systematically evaluated by CT scan of chest and abdomen at baseline and at weeks 8, 16 and 24 (end of treatment). All imaging was reviewed at the multidisciplinary tumor board and patients were considered for resection, RFA or SBRT if eligible. After completion of treatment a follow-up schedule of CT scan, clinical exam and CEA measurements were done every three months. At time of progression, patients were considered for all available standard treatment options.
All chemo-embolizations were done by experienced invasive radiologist using a femoral approach catheterization of the hepatic artery in local anesthesia. The vascular anatomy was identified by angiogram prior to the delivery of the irinotecan loaded beads of $70-150 \mu \mathrm{m}$ in diameter. A microcatheter was placed in the tumor feeding artery if possible. Otherwise in the left or right hepatic artery, peripherally to extrahepatic branches. The embolic procedure was given with a solution of $5 \mathrm{mg}$ of irinotecan $\mathrm{pr} \mathrm{ml}$ in repeated doses until a maximum of $40 \mathrm{ml}$ were administered or until a decreased flow was recognized. Injection of solution was monitored under fluoroscopy to ensure forward flow. Occlusion of tumor feeding artery was avoided.

Patients were hospitalized during the DEBIRI-TACE procedure, while standard systemic chemotherapy was given in out-patient clinic. According to protocol, a regimen of supportive care was applied consisting of anti-emetics (Ondanstron $8 \mathrm{mg}$, emperal $20 \mathrm{mg}$, methylprednisolone $80 \mathrm{mg}$ ), antibiotics (piperacillin/tazobactam 4/0.5 g $1 \mathrm{~h}$ prior to embolization), analgesics (morphine $10 \mathrm{mg}$ ) and sedative (diazepam $10 \mathrm{mg}$ ). Patients received hydration with saline and were monitored with electrocardiogram, pulsoximetri and blood pressure measurements.

Outcome. The primary endpoint of this study was response rate, and secondary endpoints were survival, toxicity and translational research. Response evaluation was done using the Response Evaluation Criteria in Solid Tumors (RESCIST 1.1) (16) classifying patients as either a complete response (CR), partial response (PR), stable disease (SD) or progressive disease (PD). The best obtained response allowed for calculation of the response rate. Patients were classified as No Evidence of Disease (NED) if no visible residual disease was detected on a CT scan.

Toxicity and adverse events (AE) were registered prospectively during the trial and recorded using the Common Terminology Criteria for Adverse Events (CTC) v3.0. (17). Pain was further quantified by the visual analogue score (VAS) (18) ranging from 0-10.

Biomarkers. Blood samples for translational use were drawn at baseline, prior to each DEBIRI-TACE, $24 \mathrm{~h}$ post-embolization (concurrent with mFOLFOX6) and prior to each subsequent cycle of protocol treatment.

Analysis of cfDNA was done using a fluorescent assay for cfDNA quatificantion as originally described by Goldshtein et al (19) and modified by our group (20). In brief, $40 \mu 1$ plasma we used, adding $\mathrm{SYBR}^{\circledR}$ Gold Nucleic Acid Gel Stain $(1: 8,000)$. The quantification of fluorescence was performed with a 96-well fluorometer (Infinite F200 PRO; Tecan Group, Ltd., Mannedorf, Switzerland) at an emission wavelength of $535 \mathrm{~nm}$ and an excitation wavelength of $485 \mathrm{~nm}$ in a black 96-well plate (Bio-Plex Pro Flat Bottom Plates; Bio-Rad Laboratories, Inc., Hercules, CA, USA).

DNA standards were prepared from Human Control Genomic DNA (Thermo Fisher Scientific, Inc., Waltham, MA, USA) diluted with a $10 \%$ bovine serum albumin (BSA; Sigma-Aldrich; Merck KGaA, Darmstadt, Germany) solution. The concentrations of the samples were calculated from a standard curve. 
For determining the definitive concentration of cfDNA in each sample, we calculated the median value of four measurements, removing outliers according to Dixons q-test if the standard deviation exceeded $10 \%$.

Statistics. Descriptive statistics were applied reporting categorical variables as counts and percentages and continuous variables by median values and $95 \%$ confidence interval (95\% CI).

For analysis of survival we used the Kaplan-Meier method and due to the small sample size $(n=14)$ we used descriptive statistics. Survival was calculated from the date of inclusion until death or censoring at end of follow-up (September 2017). Progression free survival (PFS) was defined as time until death, progression or censoring. For analysis of the prognostic value of the baseline cfDNA we stratified patients by the 75th quartile. For analysis of the cfDNA change during the 4 DEBIRI-TACE procedures we only included those patients who completed all 4 scheduled treatments $(n=11)$.

For analysis of contingency tables, we applied Fischer's exact test due to the small sample size. Comparison of continuous variables was done by linear regression analysis. $\mathrm{P}<0.05$ was considered to indicate a statistically significant difference.

All calculations and data management were performed using STATA version 14.1 (StataCorp LP, College Station, TX, USA).

\section{Results}

Patients and treatment. Fifteen patients were included, following the exclusion of one patients due to a protocol violation (9 days after inclusion), 14 patients underwent treatment and a median follow-up of 1.7 years (95\% CI: 0.5-2.9 years). The study population consisted of 8 males and the median age was 66 (range 43-74 years). Nine patients had a colonic primary cancer and the median number of liver metastases at baseline was 10 (range 1-60) with a median size of $55 \mathrm{~cm}$ (95\% CI: $39-92 \mathrm{~cm}$ ). Details on baseline characteristics are displayed in Table I. A total of 49 chemoembolization procedures were performed with a mean of 3.5 treatments pr patient. The median total dose of irinotecan embolized pr patient was $345 \mathrm{mg}$ (95\% CI: 243-395 mg). Eleven patients received the scheduled 4 DEBIRI/FOLFOX treatments while 7 patients completed the post-DEBIRI/FOLFOX planned systemic chemotherapy with FLIRI + bevacizumab.

Outcome. Among the 14 patients in this cohort the best obtained response during treatment was PR $(n=7)$, and SD $(n=4)$, while the remaining patients $(n=3)$ were not assessable for response evaluation due to failure to complete the scheduled CT scans (withdrawal of consent $n=2$, dead $n=1$ ). The corresponding response rate according to RECIST 1.1 was $50 \%$. Despite early response to treatment 2 patients developed radiological $\mathrm{PD}$ during the treatment course. Six patients were converted to eligibility for liver resection and/or RFA and 2 patients achieved status of NED (Table II).

The median progression free survival (PFS) and overall survival (OS) of all patients $(n=14)$ were 240 days $(95 \% \mathrm{CI}$ : 161-357) and 522 days (95\% CI: 174-1054) respectively, and the Kaplan-Meier curves are displayed for OS in Fig. 1 and PFS in
Fig. 2. By the end of follow-up (September 1,2017) 13 patients had died and the patient remaining alive was censored.

Toxicity. A total of $187 \mathrm{AE}$ (all grades) were documented and $25 \mathrm{AE}$ of grade 3 or higher (Table III). Twelve patients $(86 \%)$ experienced a minimum of grade 3 toxicity. The most common grade 3 or higher toxicities were nausea/vomiting, fatigue, diarrhea and pain. Three patients experienced severe organ specific complications (pancreatitis $n=1$, bowel perforation $n=1$ and kidney failure $n=1$ ). One treatment related death was recorded (kidney failure).

The median VAS score for patients was 7.5 (95\% CI: 3.7-9.0). The median dose of supplemented extra orally administered morphine was $45 \mathrm{mg}$ (95\% CI: 20-180 mg) for all completed DEBIRI TACE treatments with a wide range of 0 to $450 \mathrm{mg}$.

Biomarkers. The baseline values of CEA, LDH and cfDNA were $228 \mu \mathrm{g} / 1$ (95\% CI: 54-1,131 $\mu \mathrm{g} / \mathrm{l}), 296 \mathrm{U} / 1$ (95\% CI: $189-454 \mathrm{U} / \mathrm{l})$ and $1.10 \mathrm{ng} / \mu \mathrm{l}(95 \% \mathrm{CI}: 0.77-1.16 \mathrm{ng} / \mu \mathrm{l})$, with one missing value for the latter. A regression analysis between cfDNA and CEA respectively $\mathrm{LDH}$ demonstrate a trend for correlation in linear regression analysis for $\mathrm{LDH}(\mathrm{P}=0.06)$ and CEA ( $\mathrm{P}=0.17)$ with one outlier removed from the dataset.

Separating patients by the 75th quartile of the baseline cfDNA showed a median OS of 512 days (95\% CI: 172-870) $(n=11)$ for patients with a baseline below the 75 th quartile, and 274 days (95\% CI: $274-N A)(n=2)$ for patients above the 75 th quartile.

Examining the change in cfDNA during the 8 weeks of DEBIRI-TACE, 6 patients with a decline in cfDNA above the median value had an OS of 870 (95\% CI: 172-NA) days, while those with a cfDNA decline below the median $(n=5)$ has an OS of 362 days (95\% CI: 201-NA).

Handling the response rate as a categorical variable as PR/CR vs. not PR/CR in a $2 \times 2$ table with the median cfDNA at baseline did not show significance between response and baseline cfDNA $(\mathrm{P}=0.9)$.

Changes in the concentration of cfDNA during the treatment course were seen for all patients. All, except one, had a change in cfDNA level in the blood sample $24 \mathrm{~h}$ after the first chemo-embolization. Across all patients, the dynamic of cfDNA was more pronounced following the first chemo-embolization than the second. Later in the treatment course, the data are limited by missing blood samples post-DEBIRI and post-progression. Only six patients had blood samples available beyond four months of treatment, where one patient had an increase in cfDNA preceding the radiologic evidence of PD (patient no. 1, Fig. 3). For the remaining five patients we report a very heterogeneous pattern, although a tendency for a drop in cfDNA for patients with SD/PR. This is illustrated (Fig. 4) by the disease course for patient no. 8 where a decline in cfDNA preceded the status of NED.

\section{Discussion}

In this study we report the feasibility, efficacy and toxicity of DEBIRI-TACE/mFOLFOX6 bevacizumab as a first-line treatment for newly diagnosed mCRC. In brief, we found a response rate of $50 \%$, a median PFS of 240 days (8 months) 
Table I. Patient characteristics.

\section{Characteristics}

Sex

Male

Female

Age (years)

Median

Range

T stage

T1

$\mathrm{T} 2$

T3

$\mathrm{T} 4$

TX

$\mathrm{N}$ stage

N0

N1

$\mathrm{N} 2$

NX

Site of primary

Colon

Rectal

Number of livermets (baseline)

Median

Range

Size of largest livermet (baseline, $\mathrm{cm}$ )

Median (95\% CI)

Range (cm)

RAS/BRAF mutation

Mutation

Wild-type

Unknown

Dose irinotecan (mg)

Median (95\% CI)

Debut of metastases

Synchronous

Metachronous

Number of embolization

Median

Range
Number (\%), n=14

cfDNAng/ $\mu \mathrm{l}(95 \% \mathrm{CI})$ baseline, median

8 (57)

$6(43)$

$1.15(1.04-1.37)$

$0.78(0.64-1.11)$

66

43-74

$0(0)$

$0(0)$

4 (29)

8 (57)

2 (14)

1.15 (1.13-1.16)

$0.99(0.65-1.30)$

0.97 (0.8-1.13)

1 (7)

$1.01(1.01-1.01)$

$1.13(0.75-1.43)$

$1.06(0.64-1.24)$

4 (29)

$1.11(0.65-1.15)$

9 (64)

$1.10(0.75-1.23)$

1.07 (0.65-1.16)

10

1-60

55 (39-92)

2.5-18

\section{4}

9

1

$0.88(0.64-1.15)$

1.13 (0.86-1.30)

0.8

345 (243-395)

11 (79)

1.16 (1.01-1.43)

$1.03(0.68-1.14)$

T, T-stage of primary; N, N-stage of primary; CI, confidence interval; cfDNA, cell free DNA; RAS/BRAF, RAS/BRAF oncogene status in archival tissue.

and a median OS of 522 days (17 months), which are outcome measures comparable to other studies of DEBIRI-TACE in the first line setting $(7,8,21,22)$. Six patients were converted to eligibility for liver resection and/or RFA and 2 patients achieved status of NED, but relapsed post-study. Among them, the only patient who was still alive at end of follow-up.
In this study, the interpretation of both the clinical outcome and the corresponding translational research with cfDNA measurements are limited by the small sample size due to the early closure after inclusion only 15 of the planned 50 patients. During the trial, we saw an unexpected high level of toxicity, complications and failure to complete the scheduled treatment 
Table II. Response obtained at the CT scan at 8 weeks (post-DEBIRI-TACE) and the best obtain response during the 6 months of scheduled treatment with CT scans at week 8,16 and 24 .

\begin{tabular}{lcc}
\hline & $\begin{array}{c}\text { CT at week 8 } \\
\text { (post DEBIRI-TACE) }\end{array}$ & $\begin{array}{c}\text { Best obtained } \\
\text { response }\end{array}$ \\
\hline PD & 0 & 0 \\
SD & 9 & 4 \\
PR & 2 & 7 \\
CR & 0 & 0 \\
NA & 3 & 3 \\
Conversion to eligibility & 0 & 6 \\
for RFA/resection & & \\
Obtained status of NED & 0 & 2 \\
\hline
\end{tabular}

$\mathrm{PD}$, progressive disease; $\mathrm{SD}$, stable disease; $\mathrm{PR}$, partial regression; $\mathrm{CR}$, complete regression; NA, not assessable; NED, No evidence of disease; RFA, radio frequency ablation; CT, computed tomography.

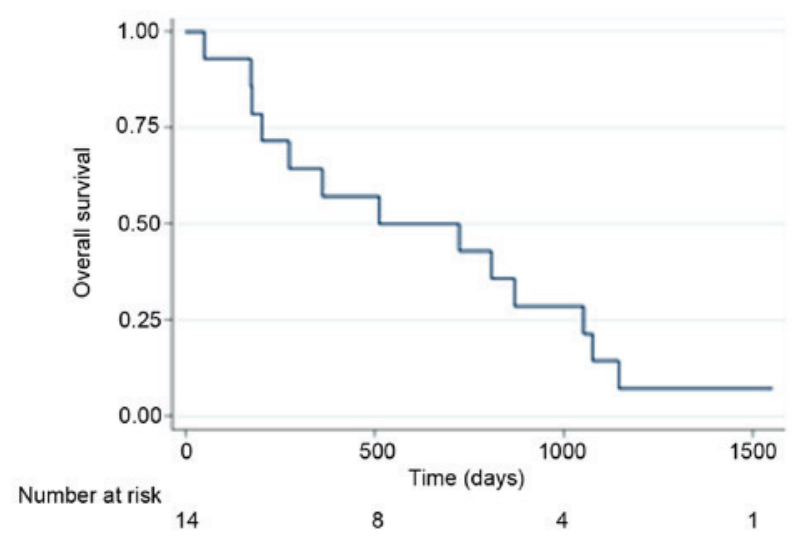

Figure 1. Kaplan-Meier curve showing the overall survival of all patients $(\mathrm{n}=14)$ from time of inclusion.

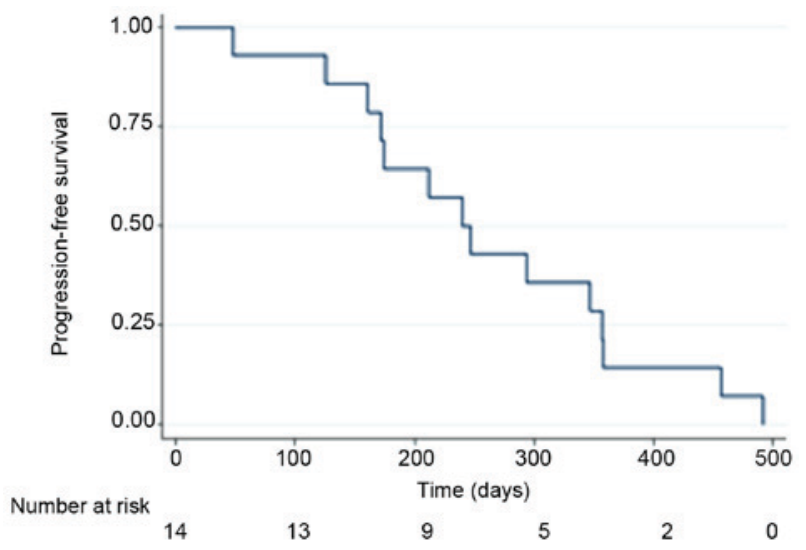

Figure 2. Kaplan-Meier curve showing the progression free survival of all patients $(n=14)$ from time of inclusion.

course of 6 months. These difficulties are not reported to the same degree in the aforementioned studies $(7,8,21,22)$ where DEBIRI-TACE is reported as a safe and well tolerated treatment.
Table III. Toxicity of grade 3 or higher.

Number of patients developing

Toxicity a grade $3-5$ toxicity $\mathrm{N}(\%)$

\begin{tabular}{ll}
\hline Vomiting & $3(21)$ \\
Fatigue & $8(57)$ \\
Nausea & $3(21)$ \\
Pain & $4(29)$ \\
Diarhea & $4(28)$ \\
Bowel perforation & $1(7)$ \\
Kidney failure & $1(7)$ \\
Infection (non neutropenic) & $1(7)$ \\
\hline
\end{tabular}

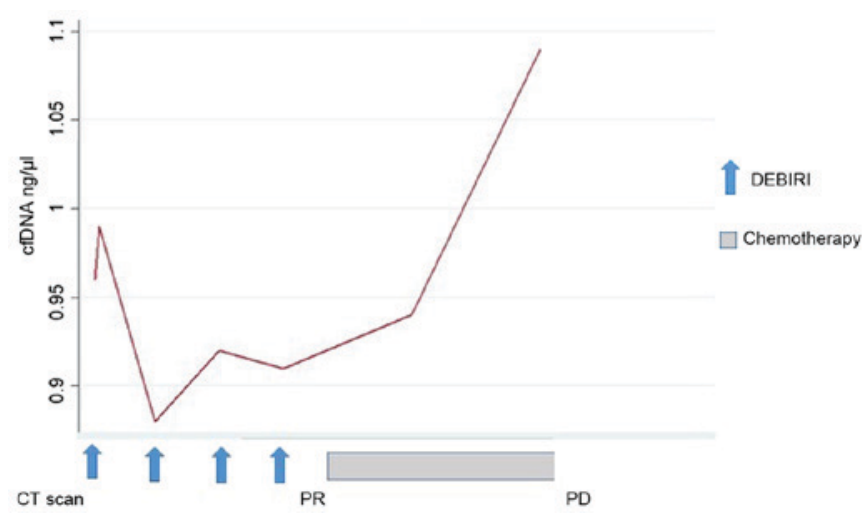

Figure 3. Treatment course for patient \#1 showing the dynamics of cell-free DNA (cfDNA) and corresponding response on imaging (CT scan). This patient had a sharp increase in cfDNA prior to the radiological diagnosis of progressive disease (PD).

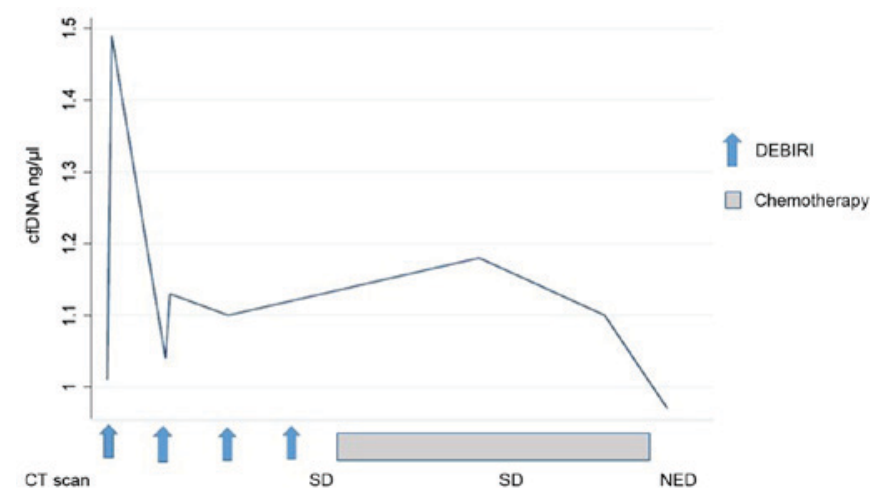

Figure 4. Treatment course for patient \#8 and corresponding level of cell-free DNA (cfDNA). A peak of cfDNA was noted following the first 2 embolizations. Subsequently a rapid decline in cfDNA prior to status of No Evidence of Disease (NED) was indicated.

It is a hypothesis, that the challenges encountered in our study is due to the intense treatment of combining DEBIRI-TACE with systemic combinations chemotherapy of mFOLFOX6 + bevacizumab, which in itself can be a toxic treatment. This combination was also examined by Martin et al (9) who randomized 60 patients between DEBIRI-FOLFOX-bevacizumab vs. DEBIRI alone, and found an increased response rate and PFS in the first group. Furthermore, they found toxicity 
of grade 3 or higher for $80 \%$ of the patients in the combination arm and for $60 \%$ in the DEBIRI only-arm. It should be noted, that the treatment scheme in this study was different with one week of separation between DEBIRI and FOLFOX, hence FOLFOX on days 0 and 14, and DEBIRI-TACE on days 7 and 21. The intensity of DEBIRI-TACE and adjacent FOLFOX $24 \mathrm{~h}$ after have not been reported previously. The most frequent grade 3/4 toxicities encounter in our study were nausea, vomiting and diarrhea, which could be attributed to the systemic exposure.

A growing body of evidence support an intense first-line treatment for $\mathrm{mCRC}$, inspired by the promising results of the Tribe study (23) using a purely systemic triplet combination of 5-fluorouracil, oxaliplatin, irinotecan (FOLFOXIRI) + bevacizumab yielding an OS of 31 months in the experimental arm. Comparison of survival between studies should be done with caution, although it is notable, with the short median survival of 17 months in this study, with the exposure to the same cytotoxic agents as in the Tribe study. In contrast to Tribe, the patients in our study all had liver limited disease, yet with a high burden of disease by number and size of metastases and baseline CEA.

Using purely descriptive statistics, we report a tendency for a shorter OS for patients with a baseline level of cfDNA above the 75th quartile. With the small sample size, we are not able to show any statistical significance, but the observed tendency is in concordance with what has previously been shown for cfDNA and survival for patients with mCRC (15). We saw a similar survival tendency for patients with a large decline in cfDNA level during the 4 DEBIRI-TACE treatments, having a numerical longer survival than patients with only a minor change in cfDNA.

We have analyzed the concentration of the total level of circulating DNA fragments, thereby not limiting our analysis to patients with detectable mutations in the blood (e.g., $\mathrm{K}$ or N-RAS). The advantage of total cfDNA quantification is a more feasible laboratory investigation in contrast to the traditional quantitative polymerase chain reaction (qPCR) techniques also requiring DNA purification. By this newly developed direct fluorescent assay, a quantitative measurement for all patients can be obtained, including for patients where the circulating DNA do not habour specific mutations. The limitations of this approach is a potential lack further molecular detail, e.g., emerging of a new RAS mutated clone would not be recognized by this methodology.

By plotting the changes in cfDNA concentration on a time series with the corresponding response evaluation by a CT scan, it is evident that the dynamics of cfNDA warrants further investigations. Interestingly, all patients, but one, had a detectable change in cfDNA level $24 \mathrm{~h}$ after the first DEBIRI-TACE. Due to our limited sample size and missing values, we are unable to demonstrate any statistical correlation between an early peak of cfDNA and the clinical outcome. However, illustrating the cfDNA course introduces the concept of molecular lead time, with a rising cfDNA preceding the radiologic diagnosis of PD (Fig. 3; patient no. 1) and a decrease in cfDNA prior to achieving status of NED on imaging (Fig. 4; patient no. 8). Early changes in circulating DNA has been describe by Tie et al (24) who examined 53 patients receiving first-line chemotherapy and found the early changes in the tumor specific cfDNA could predict later response on imaging. A potential utility of cfDNA, either as tumor specific or as a total quantity, could be the introduction of molecular lead time in diagnosis of response or progression, as also suggested by Reinert et al (25) using a more detailed molecular analysis of tumor specific DNA.

Modern treatment of $\mathrm{mCRC}$ is based on a multidisciplinary approach including with improvements in the systemic therapy integrating antiangiogenic treatment, classical chemotherapeutic agents and anti-EGFR antibodies into a combined modality with radiotherapy, surgery and invasive loco-regional therapies. DEBIR-TACE, with or without concurrent chemotherapy, constitutes a part of the loco-regional toolbox, endorsed by the ESMO guidelines (10). We propose at potential role of DEBIR-TACE as a therapy option for highly selected patients within the framework of a clinical trial, but the intense combination of DEBIRI-TACE with FOLFOX $24 \mathrm{~h}$ after embolization does not seem as a feasible design for future studies.

In conclusion, we report the clinical outcomes using DEBIRI-TACE + mFOLFOX6 bevacizumab as a first-line treatment for liver limited $\mathrm{mCRC}$ with a response rate of $50 \%$ and a median OS of 522 days (17 months). The study was impaired by an unexpected high profile of toxicity and early closure. cfDNA analysis should be explored as biomarker in larger cohorts of patients undergoing local therapy for mCRC.

\section{Acknowledgements}

Not applicable.

\section{Funding}

This study was supported by the Danish Cancer Society, Novo Nordisk Foundation and Agnes og Ejnar Danielssens Foundation.

\section{Availability of data and materials}

The datasets used and/or analyzed during the current study are available from the corresponding author on reasonable request.

\section{Authors' contributions}

MJ, AR, FVM and DTN collaborated in the study design and conception. AKB, MJ, FVM, DTN, AR, BS and KLS were involved in the acquisition, analysis or interpretation of data. AKB, MJ, FVM, DTN, AR, BS and KLS drafted the paper or revised it critically. AKB, MJ, FVM, DTN, AR, BS and KLS approved the final manuscript.

\section{Ethics approval and consent to participate}

The Regional Ethics Committee approved the study protocol and all patients consented to inclusion.

\section{Consent for publication}

All patients included in this study have consented to publication at the time of inclusion into the protocol. 


\section{Competing interests}

The authors declare that they have no competing interests.

\section{References}

1. Ferlay J, Soerjomataram I, Dikshit R, Eser S, Mathers C, Rebelo M, Parkin DM, Forman D and Bray F: CCancer incidence and mortality worldwide: Sources, methods and major patterns in GLOBOCAN 2012. Int J Cancer 136: E359-E389, 2015.

2. Weiss L, Grundmann E, Torhorst I, Hartveit F, Moberg I, Ider M, Fenoglio-Preiser CM, Napier J, Horne CH, Lopez MJ, et al: Haematogenous metastatic patterns in colonic carcinoma: an analysis of 1541 necropsies. J Pathol 150: 195-203, 1986.

3. Kopetz S, Chang GJ, Overman MJ, Eng C, Sargent DJ, Larsson DW, Grothey A, Vauthey JN, Nagorney DM and McWilliams RR: Improved survival in metastatic colorectal cancer is associated with adoption of hepatic resection and improved chemotherapy. J Clin Oncol 27: 3677-3683, 2009.

4. Gillams AR and Lees WR: Five-year survival in 309 patients with colorectal liver metastases treated with radiofrequency ablation. Eur Radiol 19: 1206-1213, 2009.

5. Fode MM and Høyer M: Survival and prognostic factors in 321 patients treated with stereotactic body radiotherapy for oligo-metastases. Radiother Oncol 114: 155-160, 2015.

6. Yamada R, Sato M, Kawabata K, Nakatsuka H, Nakamura K and Takashima S: Hepatic artery embolization in 120 patients with unresectable hepatoma. Radiology 148: 397-401, 1983.

7. Aliberti C, Tilli M, Benea G and Fiorentini G: Trans-arterial chemoembolization (TACE) of liver metastases from colorectal cancer using irinotecan-eluting beads: Preliminary results. Anticancer Res 26: 3793-3795, 2006.

8. Fiorentini G, Aliberti C, Tilli M, Mulazzani L, Graziano F, Giodani P, Mambrini A, Montagnani F, Alessandroni P, Catalano V and Coschiera P: IIntra-arterial infusion of irinotecan-loaded drug-eluting beads (DEBIRI) versus intravenous therapy (FOLFIRI) for hepatic metastases from colorectal cancer: Final results of a phase III study. Anticancer Res 32: 1387-1395, 2012.

9. Martin RC II, Scoggins CR, Schreeder M, Rilling WS, Laing CJ, Tatum CM, Kelly LR, Garcia-Monaco RD, Sharma VR, Crocenzi TS and Strasberg SM: Randomized controlled trial of irinotecan drug-eluting beads with simultaneous FOLFOX and bevacizumab for patients with unresectable colorectal liver-limited metastasis. Cancer 121: 3649-3658, 2015.

10. van Cutsem E, Cervantes A, Adam R, Sobrero A, van Krieken JH, Aderka D, Aranda Aguilar E, Bardelli A, Benson A, Bodoky $\mathrm{G}$ et al: ESMO consensus guidelines for the management of patients with metastatic colorectal cancer. Ann Oncol 27: 1386-1422, 2016

11. Spindler KL, Appelt A, Pallisgaard N, Andersen RF, Brandslund I and Jakobsen A: Cell-free DNA in healthy individuals, noncancerous disease and strong prognostic value in colorectal cancer. Int J Cancer 135: 2984-2991, 2014.
12. Mandel $\mathrm{P}$ and Metasis $\mathrm{P}$ : Les acides nucléiques du plasma sanguin chez l'homme. C R Seances Soc Biol Fil 142: 241-243, 1948 (In Undetermined Language).

13. Leon SA, Shapiro B, Sklaroff DM and Yaros MJ. Free DNA in the serum of cancer patients and the effect of therapy. Cancer Res 37: 646-650, 1977.

14. Boysen AK, Wettergren Y, Sorensen BS, Taflin H, Gustavson B and Spindler KG: Cell-free DNA levels and correlation to stage and outcome following treatment of locally advanced rectal cancer. Tumour Biol 39: 1010428317730976, 2017.

15. Spindler KG, Boysen AK, Pallisgård N, Johansen JS, Tabernero J, Sørensen MM, Jensen BV, Hansen TF, Sefrioui D, Andersen RF, et al: Cell-free DNA in metastatic colorectal cancer: A systematic review and meta-analysis. Oncologist 22: 1049-1055, 2017.

16. Eisenhauer EA, Therasse P, Bogaerts J, Schwartz LH, Sargant D, Ford R, Dancey J, Arbuck S, Gwyther S, Mooney M, et al: New response evaluation criteria in solid tumours: Revised RECIST guideline (version 1.1). Eur J Cancer 45: 228-247, 2009.

17. Cancer Therapy Evaluation Program, Common Terminology Criteria for Adverse Events, Version 3.0, DCTD, NCI, NIH, DHHS March 31, 2003. http://ctep.cancer.gov. Accessed August 9, 2006.

18. Price DD, McGrath PA, Rafii A and Buckingham B: The validation of visual analogue scales as ratio scale measures for chronic and experimental pain. Pain 17: 45-56, 1983.

19. Goldshtein H, Hausmann MJ and Douvdevani A: A rapid direct fluorescent assay for cell-free DNA quantification in biological fluids. Ann Clin Biochem 46: 488-494, 2009.

20. Schou JV, Larsen FO, Sørensen BS, Abrates R, Boysen AK, Johansen JS, Jensen BV, Nielsen DL and Spindler KL: Circulating cell-free DNA as predictor of treatment failure after neoadjuvant chemo-radiotherapy before surgery in patients with locally advanced rectal cancer. Ann Oncol 29: 610-615, 2018.

21. Eichler K, Zangus S, Mack MG, Hammerstring R, Gruber-Rouh T, Galluc C and Vogl TJ: First human study in treatment of unresectable liver metastases from colorectal cancer with irinotecan-loaded beads (DEBIRI). Int J Oncol 41: 1213-1220, 2012.

22. Fiorentini G, Aliberti C, Turrisi G, del Conte A, Rossi S, Benea G and Giovanis P: IIntraarterial hepatic chemoembolization of liver metastases from colorectal cancer adopting irinotecan-eluting beads: Results of a phase II clinical study. In Vivo 21: 1085-1091, 2007.

23. Loupakis F, Cremolini C, Masi G, Lonardi S, Zagonel V, Salvatore L, Cortesi E, Tomasello G, Ronzoni M, Spadi R, et al: Initial therapy with FOLFOXIRI and bevacizumab for metastatic colorectal cancer. N Engl J Med 371: 1609-1618, 2014.

24. Tie J, Kinde I, Wang Y, Wong HL, Roebort J, Christie M, Tacey M, Wong R, Singh M, Karapetis CS, et al: Circulating tumor DNA as an early marker of therapeutic response in patients with metastatic colorectal cancer. Ann Oncol 26: 1715-1722, 2015.

25. Reinert T, Schøler L, Thomsen R, Tobiasen H, Vang S, Nordentoft I, Lamy P, Kannerup AS, Mortensen FV, Stribolt K, et al: Analysis of circulating tumour DNA to monitor disease burden following colorectal cancer surgery. Gut 65: 625-634, 2016. 